\title{
4-Point Resistance Measurements of Individual Bi Nanowires
}

\author{
Stephen B. Cronin ${ }^{a}$, Yu-Ming Lin ${ }^{b}$, Pratibha L. Gai , Oded Rabin ${ }^{\mathrm{f}}$, Marcie R. Black ${ }^{\mathrm{b}}$, \\ Gene Dresselhaus ${ }^{\mathrm{d}}$, and Mildred S. Dresselhaus ${ }^{\mathrm{e}}$ \\ ${ }^{a}$ Department of Physics, ${ }^{b}$ Department of Electrical Engineering and Computer Science, \\ ${ }^{\mathrm{c}}$ Department of Chemistry, ${ }^{\mathrm{d}}$ Francis Bitter Magnet Laboratory, and ${ }^{\mathrm{e}}$ on leave from the \\ Massachusetts Institute of Technology, Cambridge, MA 02139 \\ ${ }^{\mathrm{f}}$ DuPont Central Research and Development, Wilmington, DE 19880
}

\begin{abstract}
We have synthesized single crystal bismuth nanowires by pressure injecting molten $\mathrm{Bi}$ into anodic alumina templates. By varying the template fabrication conditions, nanowires with diameters ranging from 10 to $200 \mathrm{~nm}$ and lengths of $\sim 50 \mu \mathrm{m}$ can be produced. We present a scheme for measuring the resistance of a single Bi nanowire using a 4-point measurement technique. The nanowires are found to have a $7 \mathrm{~nm}$ thick oxide layer which causes very high contact resistance when electrodes are patterned on top of the nanowires. The oxide is found to be resilient to acid etching, but can be successfully reduced in high temperature hydrogen and ammonia environments. The reformation time of the oxide in air is found to be less than 1 minute. Focused ion beam milling is attempted as an alternate solution to oxide removal.
\end{abstract}

\section{INTRODUCTION}

The motivation for studying Bi nanowires is based on the unique properties of bulk Bi. First, Bi has very small effective masses, with mass components as small as $0.001 m_{e}[1]$. The small effective masses of $\mathrm{Bi}$ cause the effects of quantum confinement to be more pronounced since the energy of a quantized bound state is inversely proportional to the effective mass. Therefore, the effects of quantum confinement can be observed for nanowires of relatively large wire diameter. Second, bulk Bi has a very long mean free path, $\sim 0.4 \mathrm{~mm}$ at $4 \mathrm{~K}$ and $\sim 100 \mathrm{~nm}$ at $300 \mathrm{~K}[2]$. Since the diameters of the nanowires are much smaller than the mean free path of the electrons, we expect the wires to exhibit 1D ballistic transport. Third, the low melting point of $\mathrm{Bi}\left(271^{\circ} \mathrm{C}\right)$ allows us to prepare the wires by pressure injection of molten $\mathrm{Bi}$ into a porous alumina template. It will be shown that this fabrication method yields extremely high quality crystalline nanowires. Finally, calculations of the transport properties predict that the Bi nanowires should have a very high thermoelectric efficiency [3].

As mentioned above, the effects of quantum confinement are pronounced in Bi because of its small effective masses. The change in band structure due to quantum confinement is shown schematically in figure 1 . The dashed curves depict the band structure of bulk Bi, in which the T-point valence band overlaps with the L-point conduction band by $38 \mathrm{meV}$ [4], making bulk Bi a semimetal. Due to quantum confinement effects, the band edges split into subbands as shown by the solid curves. As the diameter decreases, the separation of the subbands gets larger, and eventually the lowest conduction subband and the highest valence 
subband no longer overlap and the material becomes an indirect-gap semiconductor. This is referred to as the semimetal-to-semiconductor transition.

Detailed calculations of the energies of the subbands as a function of wire diameter have been carried out by solving Schrödinger's equation numerically for Bi nanowires with a circular cross section [3]. The semimetal-to-semiconductor transition was found to occur at a critical diameter of $\sim 50 \mathrm{~nm}$. For most other materials, where $m^{*} \sim m_{e}$, the effects of quantum confinement in a 50nm diameter wire would be negligible. However, because of the extremely small effective masses in $\mathrm{Bi}$, the quantum effects are so pronounced that we expect to observe dramatic changes in the electronic properties of the nanowires due to this semimetal-tosemiconductor transition at relatively large wire diameters.

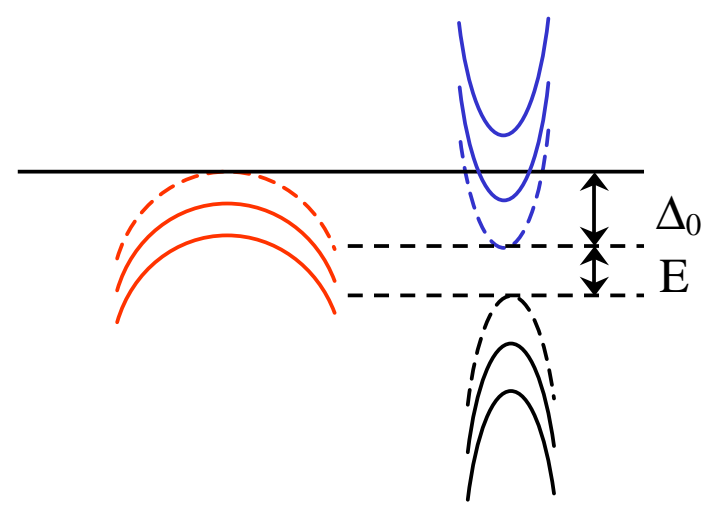

Figure 1. The quantized band structure of a Bi nanowire depicted schematically.

The Bi nanowires used in this study were prepared by a pressure injection method, whereby molten $\mathrm{Bi}$ is injected into a porous alumina template at a high pressure. Details of this fabrication process are published elsewhere [5]. We then dissolve the alumina template in a solution of $45 \mathrm{~g} / \mathrm{l} \mathrm{CrO} 3$ and 5 vol. $\% \mathrm{H}_{3} \mathrm{PO}_{4}$, leaving a solution of free standing wires.

Initial attempts were made to pattern 4-point electrodes on top of a single $\mathrm{Bi}$ nanowire using electron-beam lithography [6,7]. However, due to the very high contact resistances observed, reliable data could not be attained below room temperature. An oxide layer was suspected to be the cause of the high contact resistance observed.

Figure 2 shows a high resolution transmission electron microscope (HRTEM) image of a single Bi nanowire. As expected, we found a very thick amorphous oxide layer surrounding the crystalline Bi nanowire. For the wire in figure 2, the initial diameter was 40nm. However, after oxidation, the crystalline $\mathrm{Bi}$ part of the nanowire is reduced to $25 \mathrm{~nm}$ and is surrounded by a $7 \mathrm{~nm}$ thick oxide layer. The lattice fringes in this image indicate that the Bi nanowire has excellent single crystallinity.

The thick oxide observed in the HRTEM imaging explains the high contact resistance previously observed in the electrical measurements. Efforts were made to remove the oxide and pattern 4-point electrodes on a single Bi nanowire with low contact resistance. 


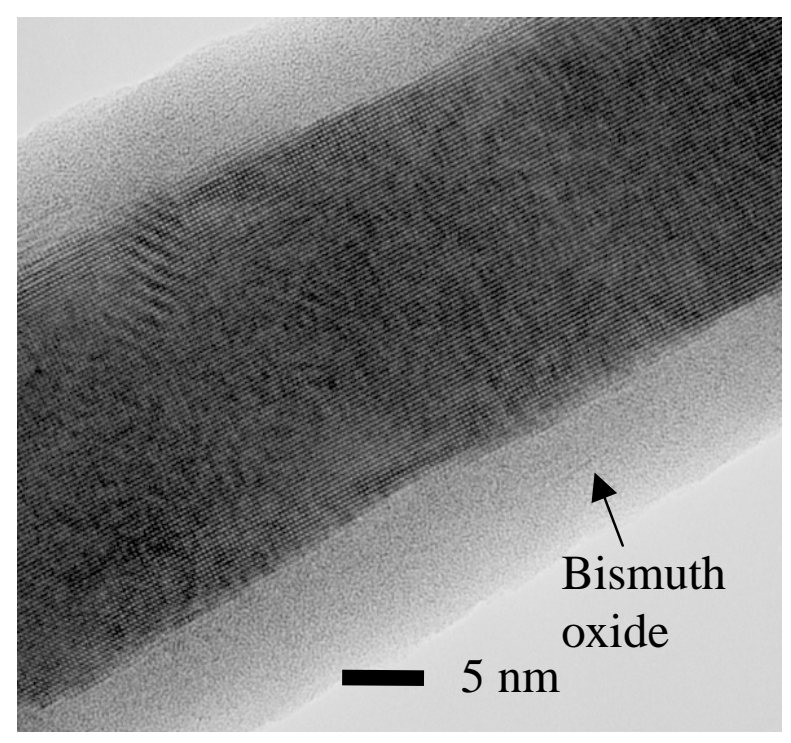

Figure 2. High resolution transmission electron microscope (HRTEM) image of a 40nm diameter Bi nanowire.

\section{OXIDE REMOVAL: Acid Etch}

As a first attempt, we tried to remove the oxide layer from the nanowire using a dilute acid. Samples were prepared for the HRTEM by putting a drop of solution containing free standing wires on a TEM grid. The grid with nanowires was then dipped in $\mathrm{HCl}$ diluted 10:1 in water for 3 seconds expecting the acid to dissolve the oxide and leave the Bi intact. However, we found that the acid dissolved the $\mathrm{Bi}$, while leaving the oxide shell intact, the opposite of our intention. Figure 3 shows the HRTEM image of the resulting bismuth-oxide nanotube after this process. Several other acids were tried, such as $\mathrm{HF}, \mathrm{H}_{2} \mathrm{SO}_{4}$ and $\mathrm{H}_{3} \mathrm{PO}_{4}$, but all yielded similar results. Despite the fact that this is an interesting result, it is not the desired result for achieving good contacts for the 4-point resistance measurement.

\section{OXIDE REMOVAL: Hydrogen Annealing}

As a second attempt to remove the oxide from the Bi nanowires we used an environmental-HRTEM (environmental-high resolution transmission electron microscope) in the DuPont Research and Development Center developed by Dr. Pratibha Gai, to study chemical reactions between gas molecules and solids directly on the atomic scale $[8,9]$. This Environmental-HRTEM system allows one to flow hydrogen gas through the sample chamber at elevated temperatures, while imaging the reaction on an atomic scale. We found that after flowing hydrogen through the sample space at $130^{\circ} \mathrm{C}$ for 6 hours, we were able to completely reduce the oxide. Figure 4 shows the HRTEM images before and after the hydrogen annealing. The top part of figure 4 a shows crystalline bismuth, below which appears the amorphous oxide layer, as indicated next to the figure. After hydrogen annealing, shown in figure $4 \mathrm{~b}$, the oxide has been successfully removed and there is a clean crystalline interface at the surface of the nanowire. 


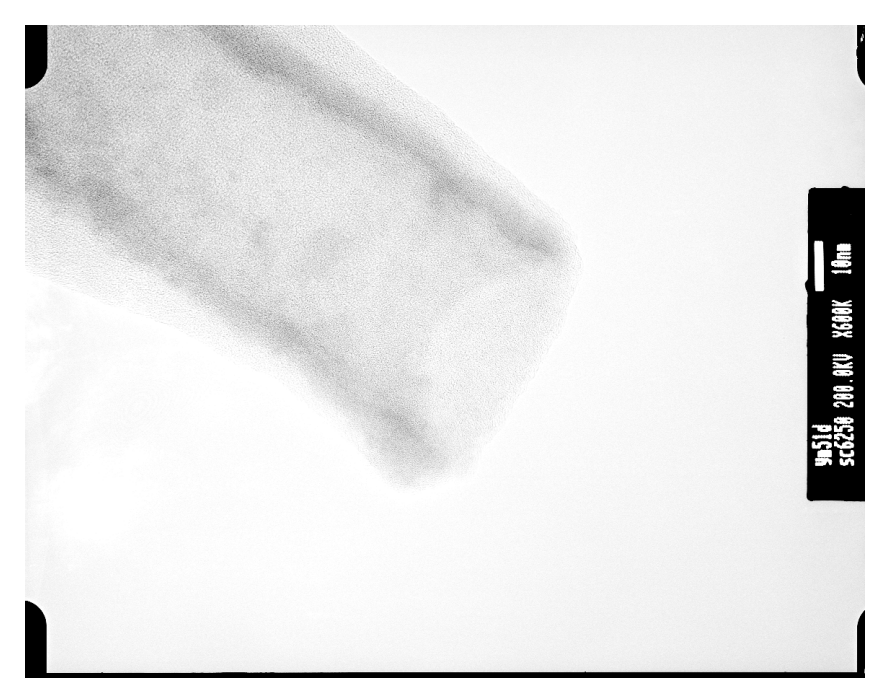

Figure 3. High resolution transmission electron microscope (HRTEM) image of an empty bismuth-oxide shell, remaining after the $\mathrm{HCl}$ acid dip.

The same result was also achieved when we repeated the experiment with ammonia gas instead of hydrogen gas.

Although we have successfully removed the oxide from the Bi nanowires, the oxide was found to reform in air in less than one minute. Therefore, in order for this technique to be applied to the 4-point resistance measurement it must be done in situ, together with the metal deposition of the 4-point electrode pattern. That is, the 6 hour hydrogen (or ammonia) anneal must be done right before the deposition of metal electrodes, while maintaining a high vacuum to prevent the oxide from reforming on the nanowire. Because of the dangers associated with hydrogen (and ammonia), this in situ oxide reduction was not performed.
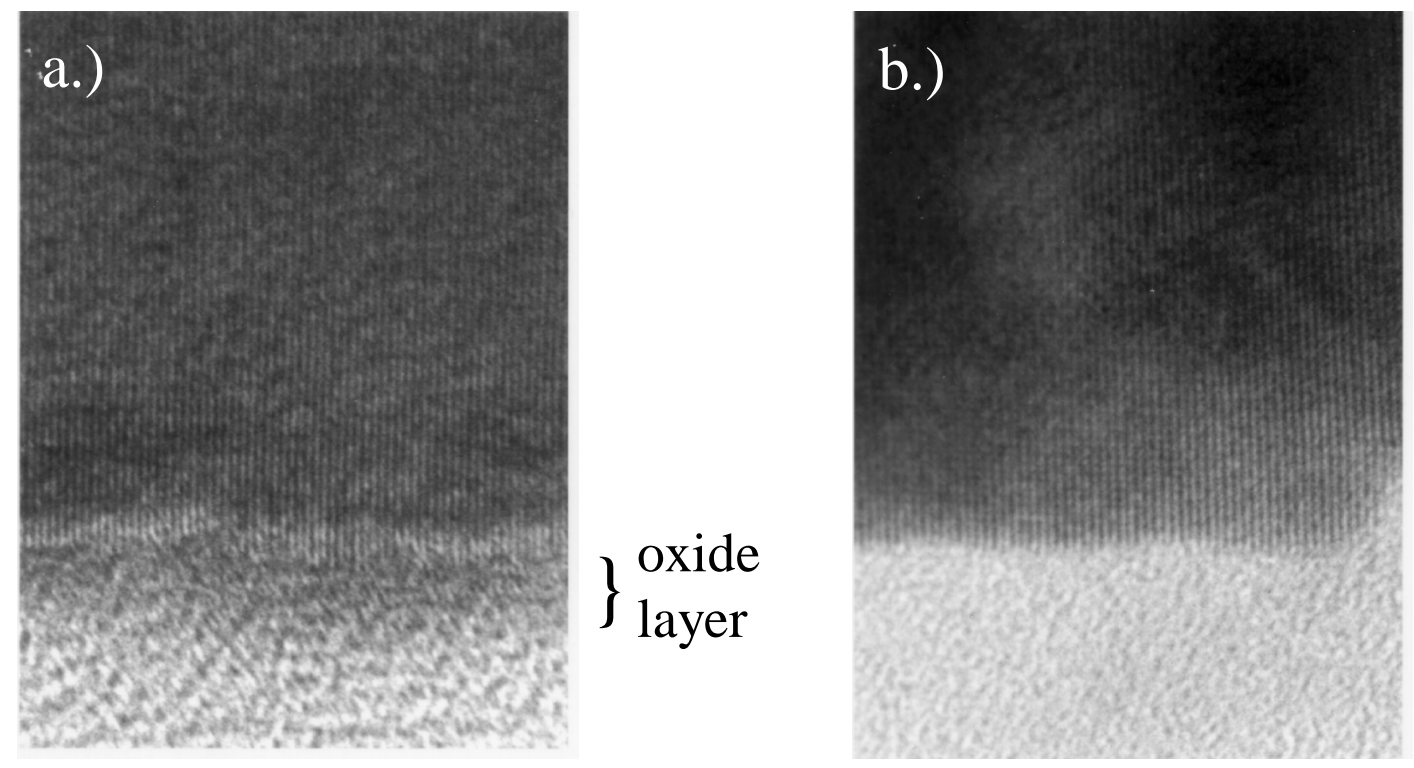

Figure 4. High resolution transmission electron microscope (HRTEM) image of a Bi nanowire a.) before and $b$.) after annealing in hydrogen gas at $130^{\circ} \mathrm{C}$ for 6 hours. 


\section{OXIDE REMOVAL: Focused Ion Beam (FIB) Milling}

As a third attempt to remove the oxide from the Bi nanowire, focused ion beam (FIB) milling was employed as a strategy to first mill away the oxide layer and then deposit platinum electrodes on the nanowire. This work was done at Harvard University in collaboration with Dr. Thomas Rueckes and Prof. Charles Lieber. The strategy is depicted schematically on the right side of figure 5 . On the left side of figure 5 the scanning electron microscope (SEM) image shows a 40nm diameter Bi nanowire with four platinum electrodes as deposited by a focused ion beam. First the nanowire was milled with a gallium ion beam in the selected areas of the 4-point electrode pattern. Then platinum was deposited to form the 4-point electrode pattern.

Since bismuth is known to be a very soft material and the bismuth-oxide is expected to be very hard, the amount a gallium ion milling is critical. If the ion beam mills beyond the surface oxide, the inner bismuth core will start to mill extremely fast, potentially depleting the entire wire. However, if the wire is insufficiently milled, the oxide will not be depleted enough to reduce the contact resistance. The contact resistance of the sample shown in figure 5 was on the order of $1 \mathrm{M} \Omega$, indicating that there was insufficient milling of the oxide to significantly reduce the contact resistance. In the future, a more systematic study of the different milling dosages will be carried out.
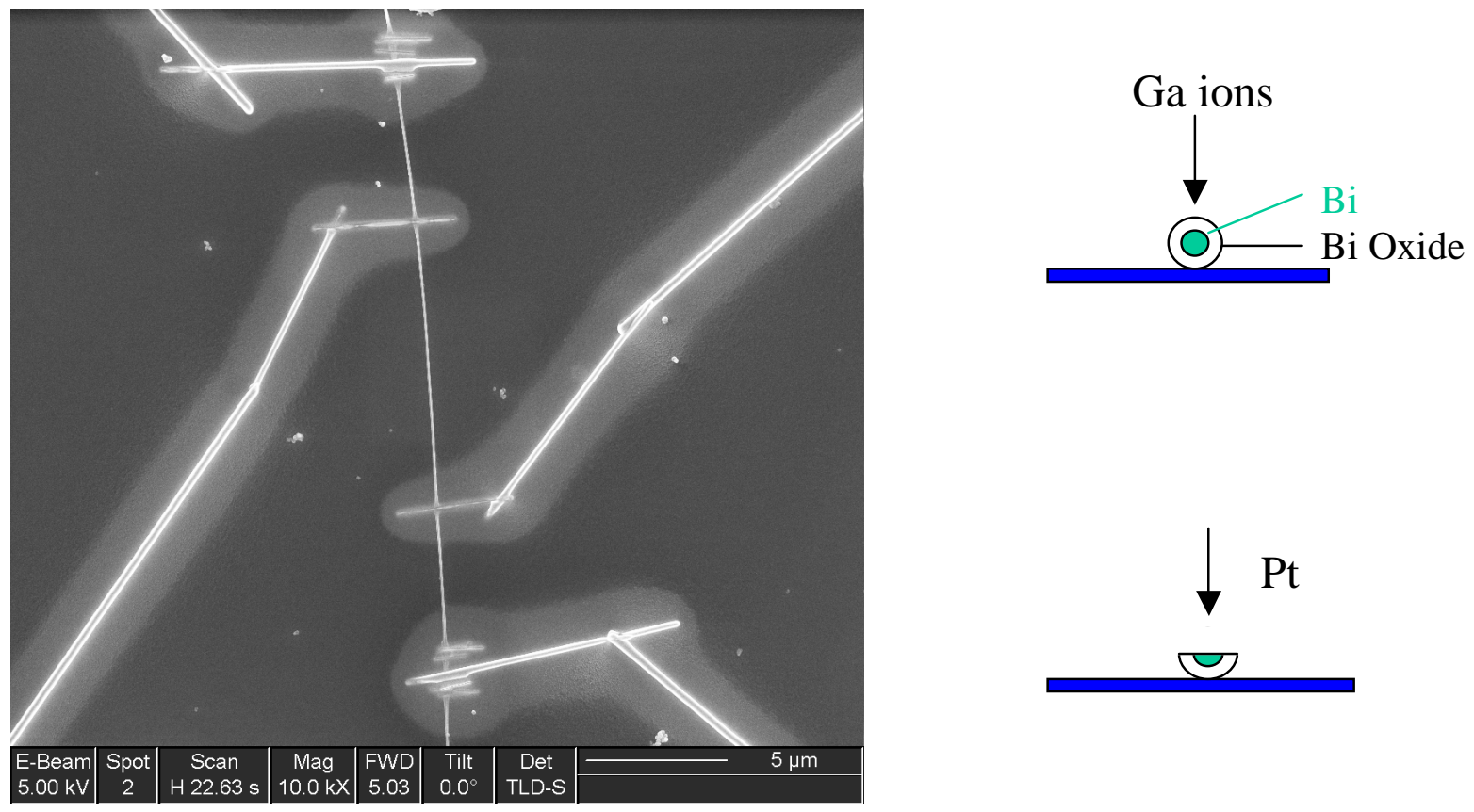

Figure 5. Right: schematic diagram depicting the two step process of gallium ion milling and platinum deposition. Left: scanning electron microscope (SEM) image of a $40 \mathrm{~nm} \mathrm{Bi}$ nanowire with 4-point Pt electrodes, prepared by first ion milling selectively the areas underneath the four electrodes. 


\section{CONCLUSION}

We have prepared single crystal $\mathrm{Bi}$ nanowires in the diameter range 10 to $200 \mathrm{~nm}$. HRTEM (high resolution transmission electron microscopy) imaging shows that a $7 \mathrm{~nm}$ thick oxide is formed on the Bi nanowires that causes very high contact resistance when electrodes are patterned on the nanowires. Further HRTEM studies show that acids dissolve the crystalline bismuth faster than the oxide, leaving an oxide nanotube. High temperature hydrogen and ammonia environments were found to reduce the oxide after a sufficient amount of time. However, this method must be used in situ with the deposition of metal because of the extremely rapid formation of the oxide in air. The focused ion beam is a promising technique for removing the oxide, however the critical milling dosage must be precisely calibrated to ensure complete sputtering of the oxide without damaging the nanowire.

\section{ACKNOWLEDGEMENTS}

The authors would like to thank Dr. Thomas Rueckes and Prof. Charles Lieber at Harvard University and also Michael Frongillo at the M.I.T. electron microscopy facility for skillful microscopy work. The authors gratefully acknowledge support from ONR under MURI subcontract \#205-G-7A114-01 and the US Navy under contract N00167-98-K-0024.

\section{REFERENCES}

1. R.T. Isaacson and G.A. Williams, Phys. Rev. 185, 682 (1969).

2. R. Hartman, Phys. Rev. 181, 1070 (1969).

3. Y.-M. Lin, X. Sun, M.S. Dresselhaus, Phys. Rev. B, 62, 4610 (2000).

4. C.F. Gallo, B.S. Chandrasekhar, and P.H. Sutter, J. Appl. Phys. 34, 144 (1963).

5. Z.Zhang, D. Gekhtman, M.S. Dresselhaus, and J.Y. Ying, Chem. Mater. 11, 1659 (1999).

6. S.B. Cronin, S. B. Cronin, Y.-M. Lin, T. Koga, J. Y. Ying, and M. S. Dresselhaus, "Transport Measurements of Individual Bismuth Nanowires", in Molecular Electronics: MRS Symposium, edited by S.T. Pantelides, et. al., Materials Research Society Press, Pittsburgh, PA, December, 1999.

7. S. Cronin, Y.-M. Lin, T. Koga, X. Sun, J. Y. Ying, and M. S. Dresselhaus, "Thermoelectric Investigation of Bismuth Nanowires", in The $18^{\text {th }}$ International Conference on Thermoelectrics: Symposium Proceedings, Baltimore, MD, 1999.

8. P.L. Gai: Direct Probing of Gas Molecules-Solid Catalyst Interactions On the Atomic Scale; Adv. Materials., 10, 1259 (1998).

9. P.L. Gai: Environmental-HREM of Gas-Catalyst Reactions, Topics in Catalysis, 8, 97 (1999). 